\title{
Enantioselective Chromatography: From its Emergence to its Successful Implementation in the Pharmaceutical Environment
}

\author{
Eric Francotte*
}

\begin{abstract}
The author describes the emergence and development of enantioselective chromatography as a powerful tool for the separation of stereoisomers, on analytical, preparative, and production scale. He summarizes his early contribution to the advance and propagation of this particular technology at the interface between chemistry, separation sciences, and physics, going back to his growing interest in chirality which was stimulated by a postdoctoral stay at the University of Geneva.
\end{abstract}

Keywords: Chiral phases · Chirality · Enantioselective chromatography

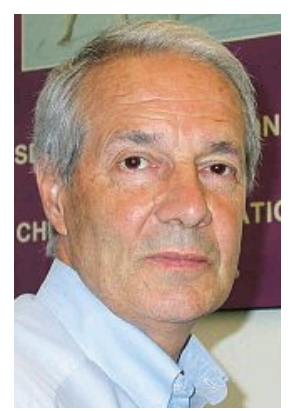

\section{Introduction}

Chirality in chemistry, biology, material science and spectroscopy has been a major topic of interest and research over the past 30 years. Switzerland contributed considerably to the development of this particular domain of research and was already early recognized as a 'chirality center' in the world as attested by the Nobel Price awarded to Vladimir Prelog in 1975. This research topic has also been a long tradition at the University of Geneva considering the pioneering works of Prof. Wolfgang Oppolzer who elaborated powerful chiral auxiliaries and strategies for enantioselective synthesis. ${ }^{[1,2]}$ After spending two years as a postdoctoral fellow in Oppolzer's research group, the conditions were given to create

${ }^{\star}$ Correspondence: Dr. E. Francotte

Novartis Institutes for BioMedical Research

Global Discovery Chemistry

WKL-122.P.25, Postfach

$\mathrm{CH}-4002$ Basel

Tel.: + 41616962971

Fax: + 41616968663

E-mail: eric.francotte@novartis.com a favorable ground to develop my passionate interest for chirality. This could be fully realized after moving to the pharmaceutical industry which, at that time, started to become quite open to questions related to the connections between molecular chirality and biological activity.

Nowadays, the systematic investigation of the biological activity of the individual stereoisomers has become the rule for all new chiral drugs and chiral considerations are now integral parts of drug research and development and of the regulatory process. However, to reach the current level of awareness and technical capabilities to deal with questions related to the concept of chirality in chemistry and biology, there was a long road and there had been an immense effort accomplished by numerous research groups in the academic and industrial research around the world. In this context, there has been a considerable development of enantioselective synthetic methodologies, which have now reached a high degree of diversity and complexity. Simultaneously, this trend has created an intensive demand for stereoselective separation techniques and analytical assays for precise determination of the enantiomeric purity of chiral compounds.

While enantioselective chromatography has now become the method of choice for analytical determinations of enantiomeric purity, the significance of the technique on a preparative scale is also well established as a powerful alternative to supplying the pure enantiomers of bioactive compounds. ${ }^{[3-6]}$ In particular, the concomitant introduction of both efficient chiral stationary phases and efficient separation techniques (HPLC, SFC, CE, SMBC) offers possibilities which were not conceivable some years ago in the field of chromatographic separations.

\section{The Development and Application of Chiral Stationary Phases}

One of the key factors which contributed to the successful application of enantioselective chromatography as a valuable approach to separate optical isomers was incontestably the development of appropriate chiral stationary phases. Although the feasibility of separating stereoisomers by chromatography on chiral packing material had already been demonstrated, ${ }^{[7-11]}$ there was only one chiral stationary phase commercially available in 1980, when we initiated our project at the central research laboratories of former Ciba-Geigy aiming to develop new chiral polymers to be used as chiral packing material. However this chiral phase developed by Pirkle ${ }^{[10]}$ did not show the desired broad applicability which was required in a pharmaceutical environment. Given this situation, we synthesized a broad variety of chiral monomers to explore the potential of the corresponding polymers as chiral stationary phases capable of discriminating optical isomers. The chiral monomers comprised among others, diamines, diols, acrylamines, acrylesters, di-acids, styrene derivatives, $\mathrm{N}$ carboxyanhydrides (Fig. 1).

Although some success was achieved with a few of the polymers derived from these monomers, it appeared quite rapidly that cellulose triacetate (CTA) (Fig. 2) showed a high chiral recognition ability in terms of differentiating enantiomers and an exceptionally broad applicability regarding the structural diversity of the resolved racemates. 


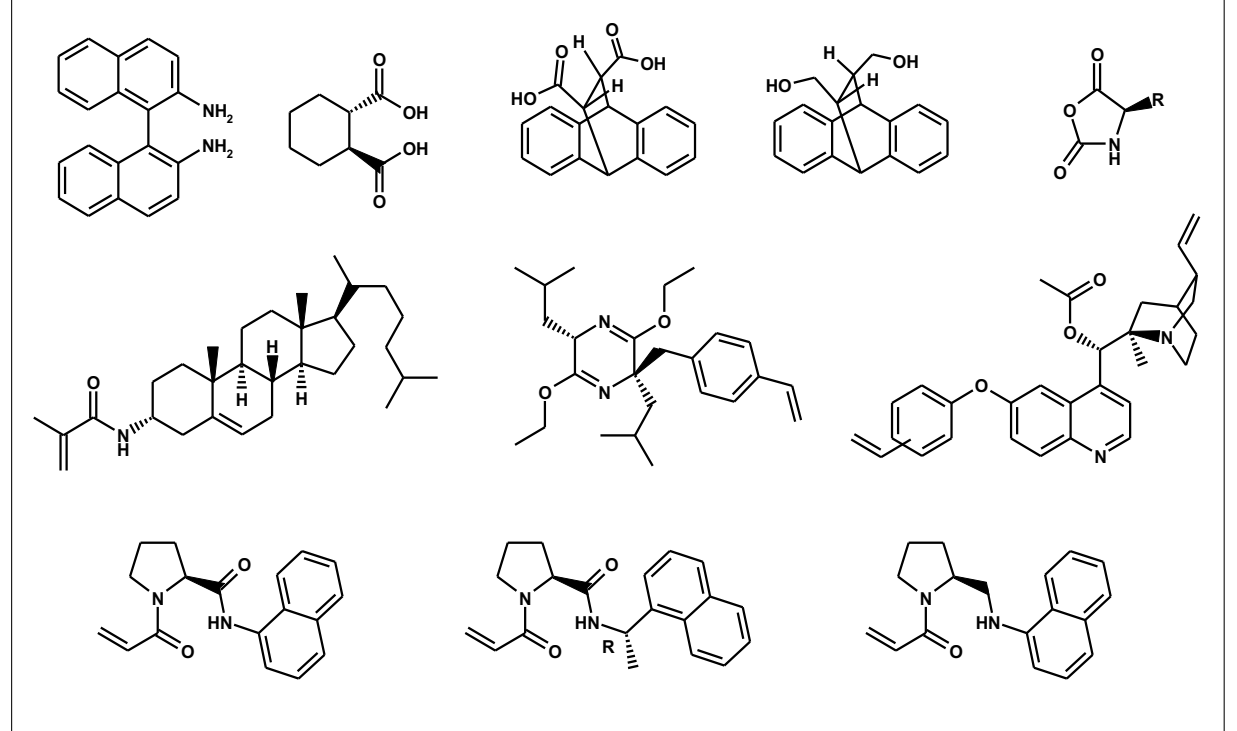

Fig. 1. Structures of selected chiral monomers synthesized for preparing chiral polymers. etry, ${ }^{[15]} \mathrm{X}$-ray, ${ }^{[16]}$ solid-state NMR or Raman spectroscopy to understand the mechanism of interaction with the respective enantiomers of racemic compounds. From these investigations, it was concluded that i) the supramolecular structure of cellulose triacetate (crystalline arrangement of the polymer chains) is a determining factor for the chiral recognition, ${ }^{[16]}$ ii) different interaction sites are probably involved in the chiral recognition process ${ }^{[17]}$ and, iii) an in silico prediction of a chiral separation with this type of polymer would be illusory as long the exact structure of the interaction sites is not clearly defined. The critical influence of the supramolecular structure of CTA on chiral recognition is shown in Fig 5. For the selected racemic compounds, it is obvious that the enantioselectivity exhibited by the crystal form I of CTA is generally superior and for three compounds the elution order is reversed,

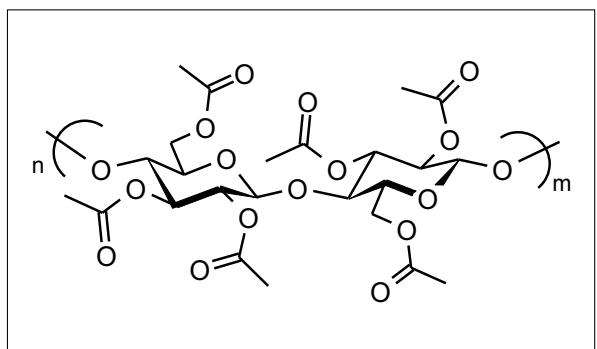

Fig. 2. Structure of cellulose triacetate (CTA).

Around $60 \%$ of the investigated chiral molecules were found to show at least an enrichment and $40 \%$ to be baseline separated. The potential of this chromatographic approach to determine the enantioselective disposition of chiral drugs directly on chiral stationary phase, without preliminary derivatization to diastereoisomers, was then rapidly recognized. This led to the first validated application in a pharmaceutical environment which was performed at former Ciba in the early eighties for the analgesic drug Oxindanac (Fig. 3). ${ }^{[12]}$

The preparative potential of the technique to produce optically pure chiral drugs in large amounts was simultaneously exploited and led, as early as 1985, to the construction of the largest chiral column ever packed with cellulose triacetate (Fig. $4)$. On this column containing $14 \mathrm{~kg}$ of cellulose triacetate, amounts of up to $150 \mathrm{~g}$ of racemic phenyldioxane for example were chromatographically resolved per run.

In order to develop improved chiral stationary phases, we started a program to investigate and determine the characteristics that made cellulose triacetate (CTA) so successful. The investigations included the application of various approaches like molecular modeling, ${ }^{[13,14]}$ microcalorim-

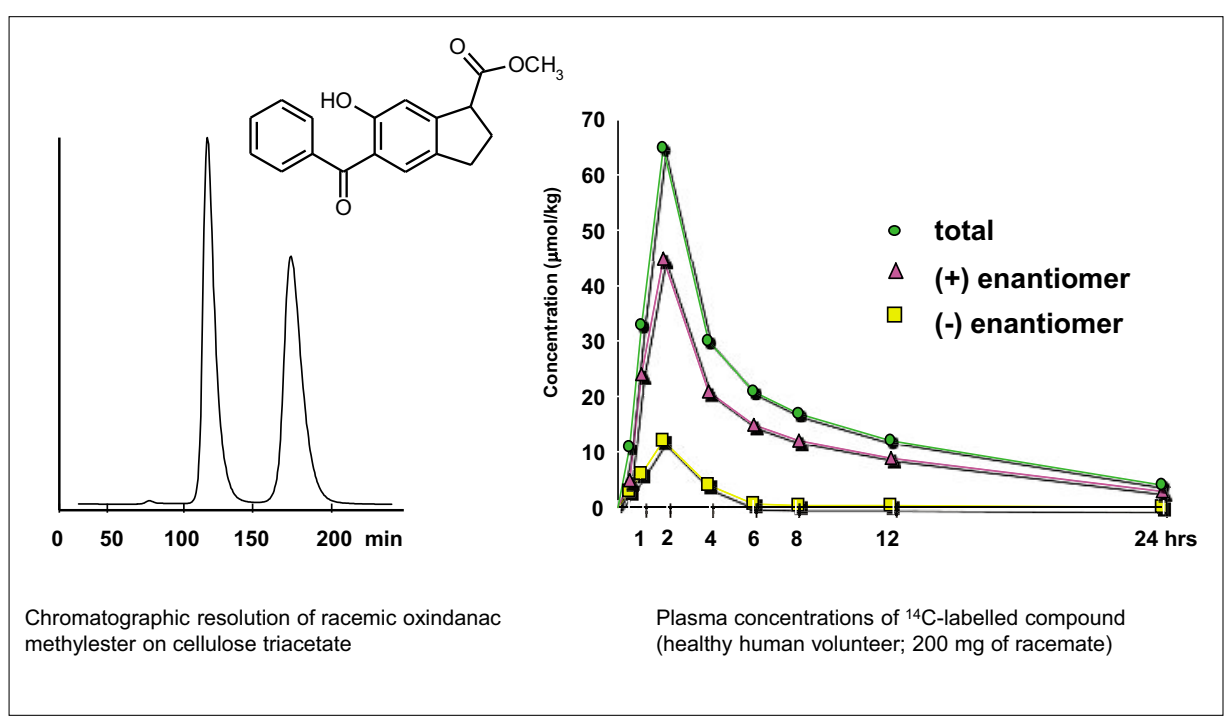

Fig. 3. Pharmacological disposition of the enantiomers of the drug Oxindanac. ${ }^{[12]}$

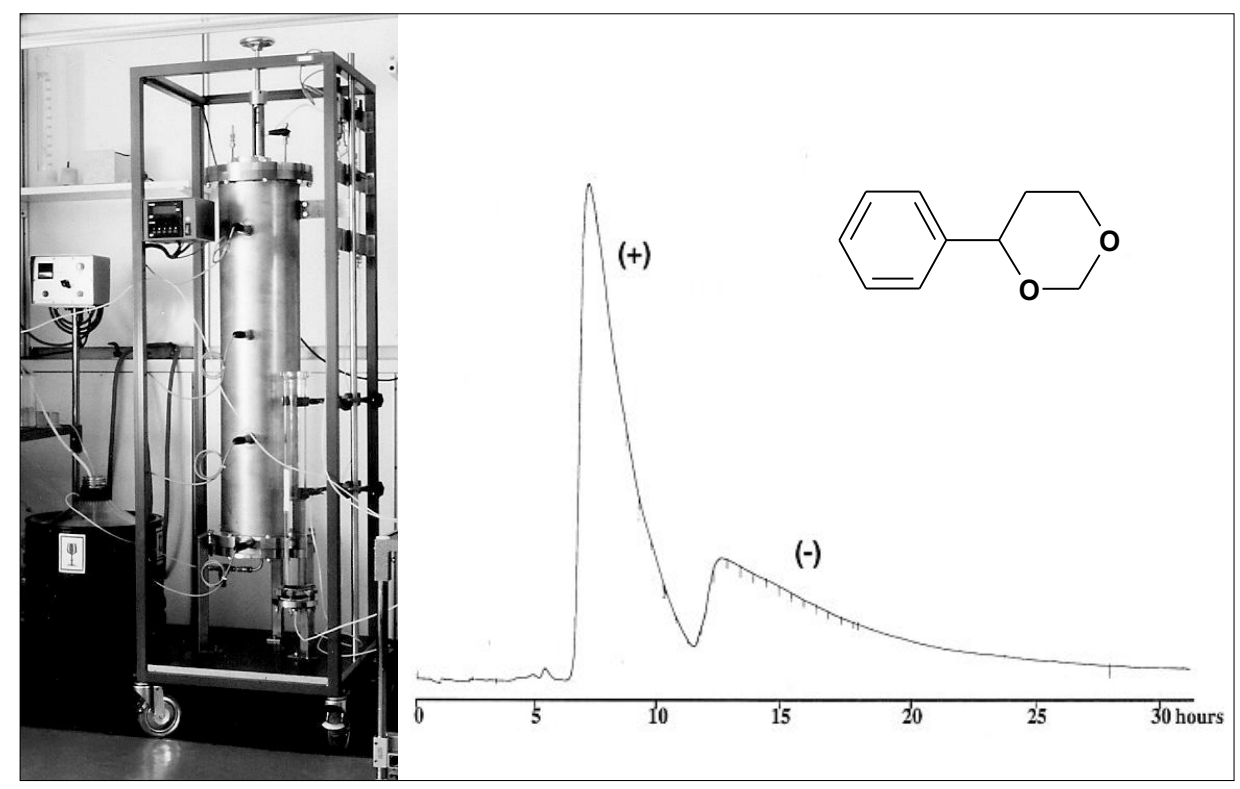

Fig. 4. Picture of the large CTA column $(20 \mathrm{~cm}$ i.d. $\times 100 \mathrm{~cm})$ and resolution of $150 \mathrm{~g}$ racemic phenyldioxane. 


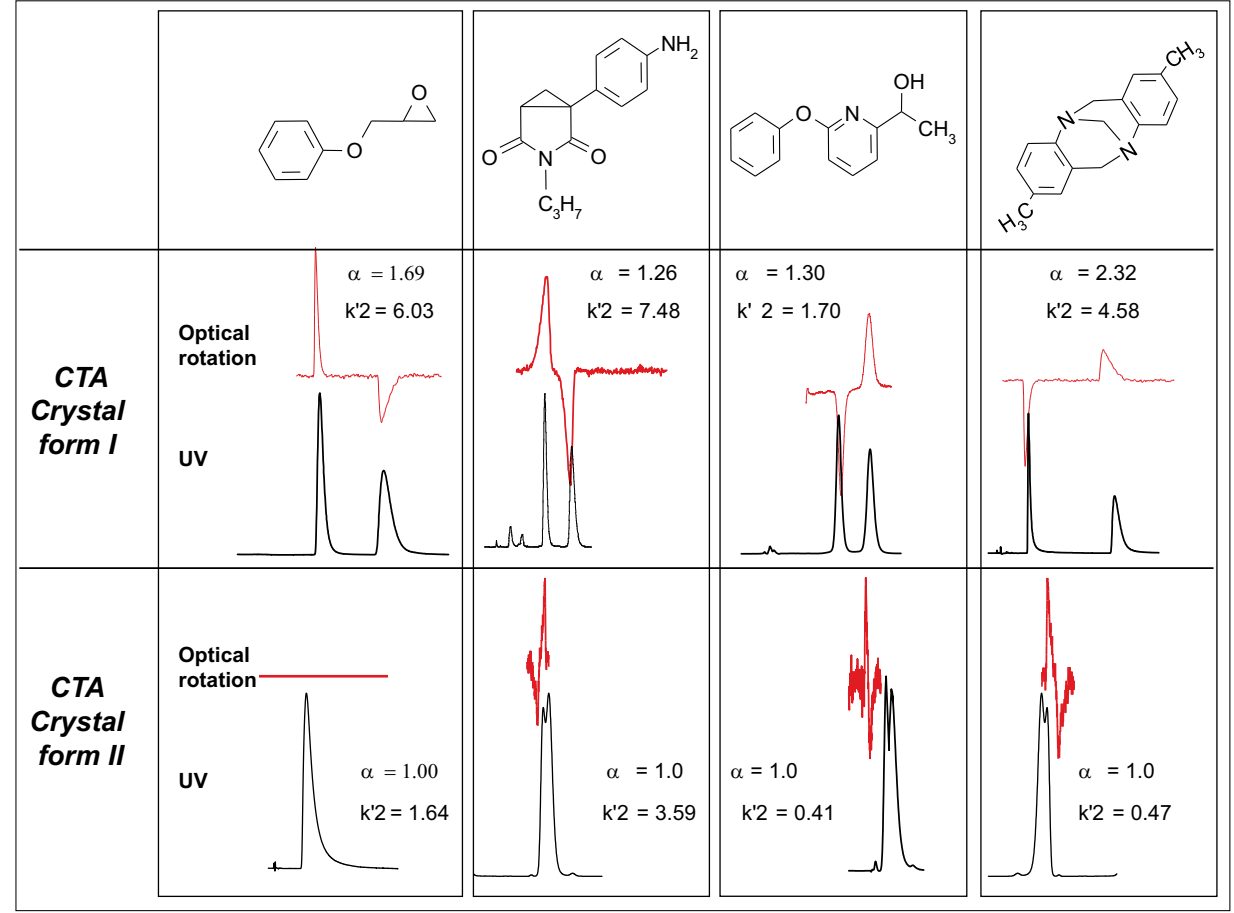

Fig. 5. Influence of the supramolecular structure of cellulose triacetate (CTA) on its chiral recognition power.

\section{Chiral carbon atom

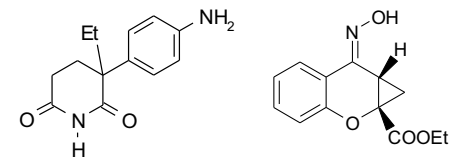

\section{Chiral nitrogen atom}

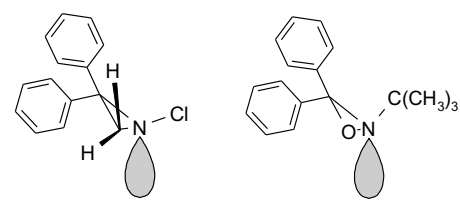

$$
\text { Chiral sulfur atom }
$$

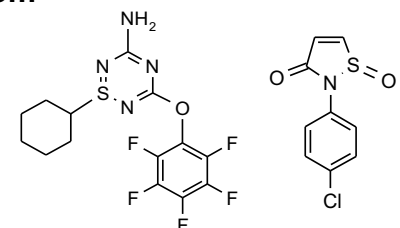

Chiral iron atom
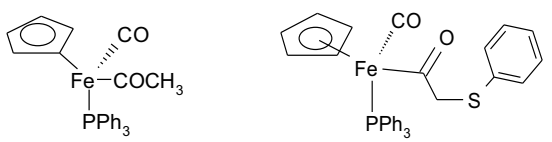

Fig. 6. Selected forms of molecular chirality which have been resolved by enantioselective chromatography in our laboratories.

although the chiral information at the molecular level is identical for both crystalline forms of CTA. ${ }^{[16]}$

Despite its relatively broad applicability and low preparation costs, CTA shows a major drawback due to its compressibility and poor mechanical stability. This problem could be overcome by the research group of Okamoto in Japan who developed a new technique to prepare a wide range of polysaccharide-based chiral stationary phases by coating silica gel with about $20 \%$ (weight) of the chiral polymers. ${ }^{[18]}$ His work confirmed the versatility and high chiral recognition ability of polysaccharides and demonstrated that this chiral recognition ability can be tuned by simply modifying the derivatization group on the hydroxyl group of the sugar moieties. ${ }^{[19]}$ Also for this kind of phase, we could show that the supramolecular structure of the polymer can be altered by varying the coating conditions, affecting the chiral recognition ability.[20] This type of phase is now widely used for analytical and preparative enantioselective chromatography. It covers almost all domains of chirality which require a precise determination of the enantiomeric composition of chiral substances (samples from enantioselective synthesis or catalysis, investigation of the stereoselectivity of biological processes in life sciences, environmental analysis, etc.) or which require the preparation of small or larger amounts of optically pure stereoisomers (drugs and pharmaceuticals, agrochemicals, chiral intermediates). Within less than 20 years, the technique has become well established and is the method of choice in all laboratories dealing with chiral molecules. Practically all kinds of molecular chirality can be discriminated by enantioselective chromatography as shown with selected examples investigated in our laboratories (Fig. 6).

By using just a limited number of chiral stationary phases (CSPs) (Fig. 7), a high success rate has already been achieved (about $90 \%$ ) as confirmed by thousands of internal applications developed at Novartis.

On a preparative scale, enantioselective chromatography is also now the first choice in the pharmaceutical industry at least during the preliminary phase of development of new chiral drug candidates, and it is routinely applied for amounts ranging from a few $\mathrm{mg}$ to several tens of grams. ${ }^{[3-6]}$ Separations of larger amounts (100 g - kilograms) are also now usually performed in the pharmaceutical industry and the technology is even already applied at the production scale. A number of optically pure drugs are now obtained by enantioselective chromatography at a scale of several metric tonnes, using the simulated moving bed technique. ${ }^{[6,21,22]}$

However, for preparative applications productivity is obviously a critical factor. Productivity depends on different parameters. Among the factors affecting productivity, the solubility of the racemate (or chiral mixture) in the mobile phase is very often a major issue when going from the analytical to the preparative scale. Due to the relatively good solubility of most polysaccharide derivatives in many common organic solvents like chlorinated alkanes, ethyl acetate, toluene, acetone, tetrahydrofuran, or dioxane, very limited options are available in terms of varying the choice of the mobile phase to improve the solubility of the racemate. All mobile phases containing these solvents or mixtures of these solvents are excluded to prevent any irreversible damage of the columns. To tackle this challenging question, 


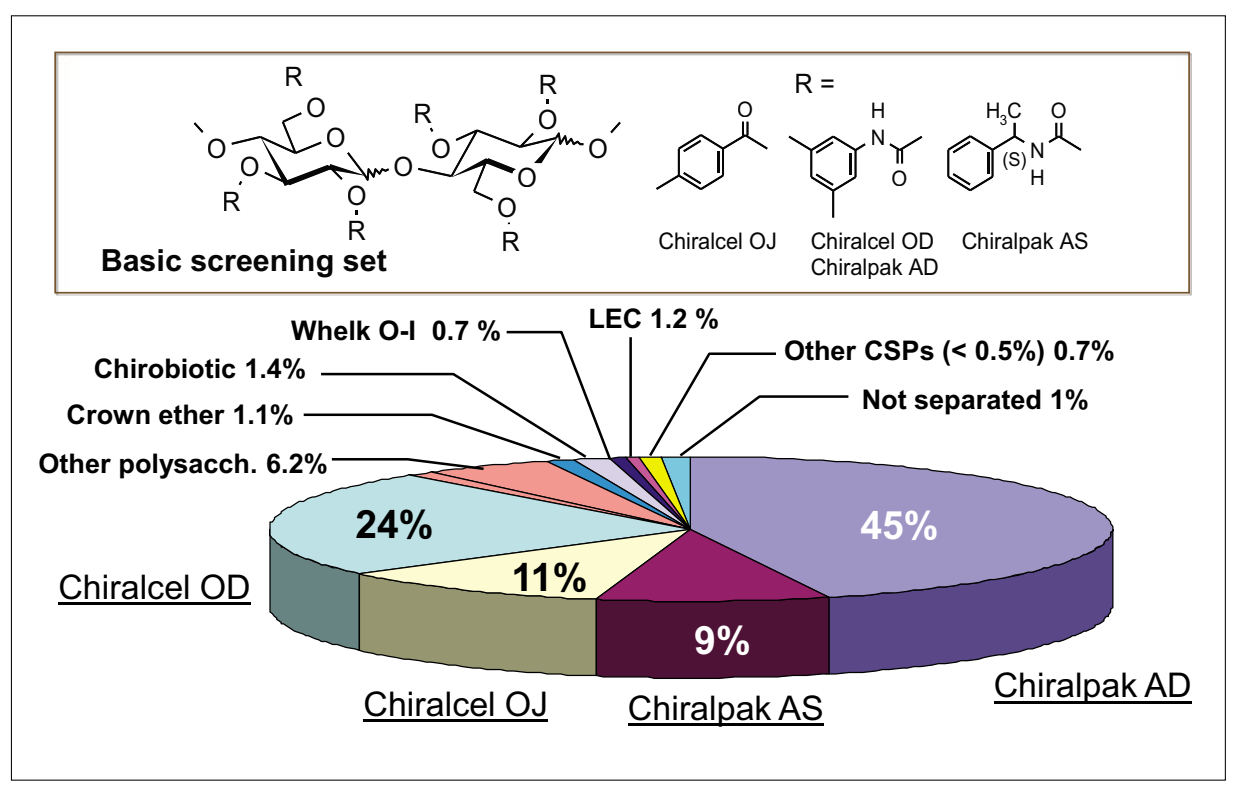

Fig. 7. Success rate for the most used CSPs (CSPs distribution for $\sim 2000$ racemic compounds).

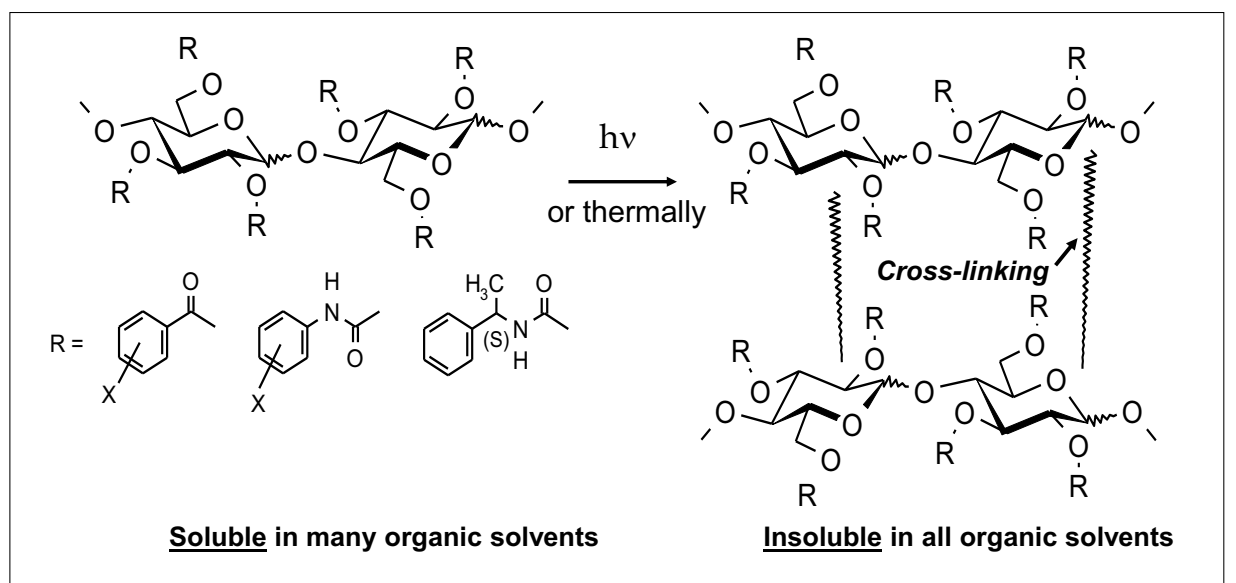

Fig. 8. Process of immobilization of polysaccharide-based CSPs.
Improved selectivity on immobilized chiral stationary phases
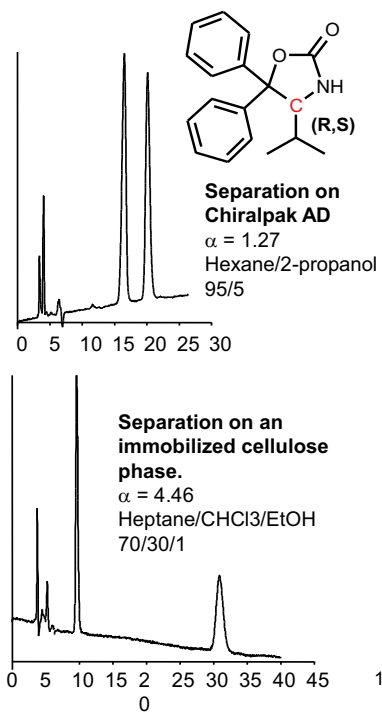

a

\section{Separation only feasible on immobilized chiral stationary phases using chlorinated solvents}

Separation on an immobilized cellulose phase Heptane/chloroform/ethano $65 / 30 / 5$

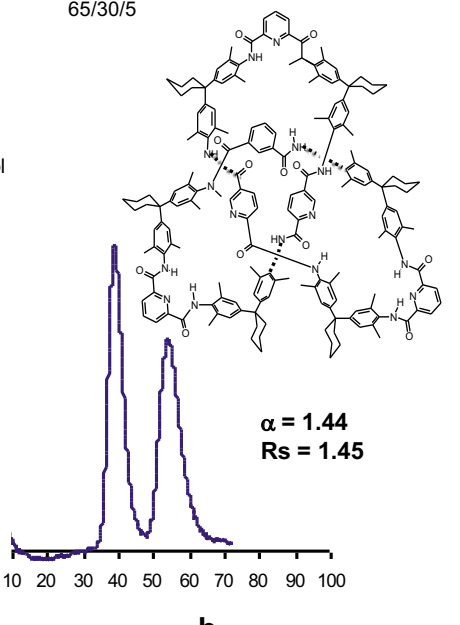

Enhanced solubility using solvents only compatible with immobilized chiral stationary phases

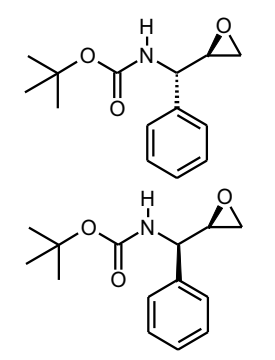

hexane/2-propanol 96/4 Solubility: $\sim 0.3 \%$

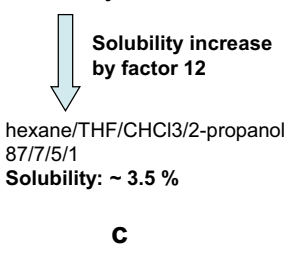

Fig. 9. Improved resolutions of chiral compounds using immobilized polysaccharide-based stationary phases. we decided to design a new generation of polysaccharide-based phases by immobilizing the chiral polymers on the silica support.

The designed process consists in exposing the coated phases to a photochemical treatment or heating in the presence of radical initiators like di-tert-butylperoxide.[23-25] By application of one of these processes, immobilization occurs presumably by cross-linking of the polysaccharide chains (Fig. 8). Once immobilized, the CSPs can be used with a large variety of mobile phases without deterioration.

A broad range of immobilized polysaccharide-based CSPs have been prepared according to the invented processes, setting up a new standard in this important field of separation sciences. Depending on the applied mobile phase, the new CSPs show that i) significant improvement of selectivity can be achieved; ii) retention time can be better adjusted; iii) considerable increase of solubility of the chiral solute can be obtained. All these features are extremely important for productivity. A series of phases produced according to this technology are now commercially available and their usefulness is confirmed by the increasing number of reported applications. In particular the immobilized chiral phase prepared from 3,5-dichlorophenyl-carbamate of cellulose shows a high potential regarding selectivity and application range. ${ }^{[26]}$ Practical use of this chiral polymer derivative in its non-immobilized form was not feasible due to the high solubility of the cellulose derivative in all non-aqueous solvent mixtures. With the new immobilized phases it is now possible to apply any kind of mobile phase, leading to improved performance in terms of enantioselectivity (Fig. 9a), of resolving poorly soluble chiral compounds as exemplified with the first resolution of chiral knots $^{[27]}$ (Fig. 9b), and of productivity due to enhanced solubility of the racemic solute (Fig. 9c).

\section{Conclusion}

Over the last 25 years, enantioselective chromatography has evolved from a basic principle to an essential tool for analyzing chiral compounds and to a powerful alternative for the preparation of optically pure compounds thanks to the development of powerful chiral stationary phases. It is now the standard technique used in all laboratories dealing with the analysis of chiral molecules. At the laboratory scale it has also become the method of choice to provide small amounts of pure stereoisomers as it is rapid, easy and generally applicable. On a pilot and process scale, the chromatographic approach ensures a 
continuous supply of optically pure substances in quantities required to perform the desired biological investigations while other preparative approaches are evaluated. Moreover, even at a production scale and especially since the introduction of the simulated moving bed technology, chromatography is now considered as one of the possible approaches for obtaining single enantiomers as demonstrated by several applications at a scale of several tonnes.

Received: October 9, 2009

[1] W. Oppolzer, Tetrahedron 1987, 43, 1969.

[2] W. Oppolzer, Angew. Chem., Int. Ed. Engl. 1984, 23, 876

[3] 'Chirality in Drug Research, Methods and Principles in Medicinal Chemistry', Vol 33, Eds. E. Francotte, W. Lindner, Wiley-VCH Verlag, Weinheim, 2006.

[4] E. Francotte, 'Preparation of drug enantiomers by chromatographic resolution on chiral stationary phases, in: The Impact of Stereochemistry on Drug Development and Use', Eds. H. Y. Aboul-Enein, I. W. Wainer Chemical Analysis Series, Vol. 142, John Wiley \& Sons, New York, 1997, pp 633-683.

[5] E. R. Francotte, J. Chromatogr. A 2001, 906, 379.

[6] 'Preparative Enantioselective Chromatography', Ed. G. Cox, Blackwell Publishing, Oxford, 2005.

[7] V. Prelog, P. Wieland, Helv. Chim. Acta 1944, 27, 1127.

[8] G. Hesse, R. Hagel, Liebigs Ann. Chem. 1976, 996.

[9] G. Blaschke, H.-P. Kraft, H. Markgraf, Chem. Ber. 1980, 113, 2318.
[10] W. H. Pirkle, T. C. Pochapsky, J. Chromatogr. 1986, 369, 175

[11] Y. Okamoto, M. Kawashima, K. Yamamoto, K. Hatada, Chem. Lett. 1984, 739.

[12] E. Francotte, H. Stierlin, J. W. Faigle, J. Chromatogr. 1985, 346, 321.

[13] R. M. Wolf, E. Francotte, D. Lohmann, J. Chem. Soc. Perkin Trans. II 1988, 893.

[14] R. M. Wolf, E. Francotte, L. Glasser, I. Simon, H. Sheraga, Macromolecules 1992, 25, 709.

[15] R. M. Wolf , E. Francotte, J. Hainmüller, Chirality 1993, 5, 538.

[16] E. Francotte, R. M. Wolf, D. Lohmann, R. Müller, J. Chromatogr. 1985, 347, 25.

[17] E. Francotte, R. M. Wolf, Chirality 1990, 2, 16

[18] Y. Okamoto, M. Kawashima, K. Hatada, J. Am. Chem. Soc. 1984, 106, 5357.

[19] Y. Okamoto, E. Yashima, Angew. Chem., Int. Ed. 1998, 37, 1021.

[20] E. Francotte, T. Zhang, J. Chromatogr. A 1995 , $718,257$.

[21] E. Francotte, P. Richert, J Chromatogr A 1997, $769,101$.

[22] 'Chirality in Drug Research, Methods and Principles in Medicinal Chemistry', Vol 33, Eds. E. Francotte, W. Lindner, Wiley-VCH Verlag, Weinheim, 2006, p. 178.

[23] E. Francotte, T. Zhang, International Patent number WO 1997/04011(06.22.1997); European Patent EP 0840752B1 (priority 21.07. 1995).

[24] E. Francotte, International Patent number WO 1997/049733(31.12.1997); European Patent EP 0907663B1 (priority 27.06.1996).

[25] E. R. Francotte, Chimia 1997, 51, 717.

[26] E. Francotte, D. Huynh, J. Pharm. Biomed. Anal. 2002, 27, 421.

[27] F. Vogtle, A. Hunten, E. Vogel, S. Buschbeck, O. Safarowsky, J. Recker, A.-H. Parham, M. Knott, W. Müller, U. Müller, Y. Okamoto, T. Kubota, W. Lindner, E. Francotte, S. Grimme, Angew. Chem., Int. Ed. 2001, 40, 2468. 